\title{
Factores biomecánicos asociados al resultado clínico de pacientes tratados con artrodesis lumbar en un centro de alta complejidad en Colombia
}

\section{Biomechanical factors related to surgical outcomes on patients treated with lumbar arthrodesis in a high complexity center in Colombia}

C. A. Lindado ${ }^{1,2}$, S. Gutiérrez $z^{3,2}$, S. Patiño $0^{4,2}$ y J. C. Acevedo ${ }^{5,2}$

${ }^{1}$ Neurocirugía, Pontificia Universidad Javeriana. Bogotá, Colombia. ${ }^{2}$ Semillero de Investigación Estudiantil del Departamento de Neurociencias. Pontificia Universidad Javeriana. Bogotá, Colombia. ${ }^{3}$ Médico y Cirujano. Pontificia Universidad Javeriana. Bogotá, Colombia. ${ }^{4}$ Estudiante de Medicina. Pontificia Universidad Javeriana. Bogotá Colombia. ${ }^{5}$ Neurocirujano. Director del Departamento de Neurociencias Hospital Universitario San Ignacio. Bogotá, Colombia

\section{ABSTRACT}

Introduction: The importance of lumbar pain management lies in its recurrence and manifestations for both the patient and society. It is necessary to establish what factors lead to success in terms of pain and functionality at the time of a lumbar arthrodesis.

Material and methods: A retrospective descriptive study was conducted that sought to determine which spinopelvic parameters and their values in terms of pelvic incidence (PI), pelvic tilt (PT), sacral slope, lumbar lordosis (LL), sagittal vertical axis (SVA), and mismatch (PI-LL) might have a potential relationship with favorable postoperative outcomes. We included 44 patients who underwent MIS spinal approaches in our center between January 2016 to December 2017. Surgical outcomes of pain and functionality were assessed using visual analogue scale and Oswestry Disability Index (ODI). Follow up was conducted through three evaluations: one before surgical intervention and 2 at 3 and 6 months postop. Due to the sample size, the authors were unable to get statistically significant results.

Results: In our descriptive retrospective study on 44 patients, we found a big positive functional change in the

Lindado CA, Gutiérrez S, Patiño S, Acevedo JC. Factores biomecánicos asociados al resultado clínico de pacientes tratados con artrodesis lumbar en un centro de alta complejidad en Colombia. Rev Soc Esp Dolor. 2020;27(3):160-167

\section{RESUMEN}

Introducción: La importancia del manejo del dolor lumbar radica en su recurrencia y en las manifestaciones tanto para el paciente como para la sociedad. Se hace necesario establecer qué factores llevan al éxito en términos de dolor y funcionalidad al momento de realizarse una artrodesis lumbar.

Materiales y métodos: Se realizó un estudio descriptivo retrospectivo que buscó determinar qué parámetros espinopélvicos de balance sagital y sus valores en términos de incidencia pélvica $(\mathrm{PI})$, inclinación pélvica (PT), inclinación sacra, lordosis lumbar (LL), eje sagital vertical (SVA) y la diferencia entre la PI y LL (PI-LL), pueden tener una potencial relación con desenlaces posquirúrgicos favorables. Se incluyeron 44 pacientes tratados en el Hospital Universitario San Ignacio entre enero de 2016 y diciembre de 2017. Se tomaron en cuenta desenlaces de dolor y funcionalidad en términos la escala visual analógica y el Oswestry Disability Index (ODI). Se realizó un seguimiento prequirúrgico, y 2 posquirúrgicos a los 3 y 6 meses. Debido al tamaño de la muestra, los autores no pudieron encontrar una significancia estadística, sin embargo, los resultados

Recibido: 22-08-2019

Aceptado: 26-01-2020

Correspondencia: Santiago Gutiérrez Gómez sgutierrezg@javeriana.edu.co 
group of patients who had a PI-LL $>10^{\circ}$ with an increase of 50 percentage points in the group of patients with good functionality (ODI: 0-20). In SVA > $5 \mathrm{~cm}$, the range of patients with good functionality had an increase of 44.6 percentage points. The range of good functionality in patients with a $\mathrm{PT}<20^{\circ}$ increased 54.1 percentage points.

Conclusions: In the present cohort, it was found that the radiological parameters that conditioned a greater percentage change in the functionality of the patients after being taken to spine surgery were SVA of base $>5 \mathrm{~cm}$ and a PI-LL base $>10^{\circ}$ and $\mathrm{PT}<20^{\circ}$.

Key words: Back pain, lumbar interbody fusion, spinopelvic parameters, spine surgery.

\section{INTRODUCCIÓN}

El dolor lumbar es un síntoma frecuente y prevalente. Un episodio de dolor lumbar agudo puede estar presente en el 75-85\% de la población general (1). En EE. UU. y otros países desarrollados, se reportan prevalencias generales del 12-13\%, prevalencia a un año del 22-65 \% y prevalencia vital del 11-84\% (2). Entre las diferentes opciones terapéuticas se encuentra la artrodesis lumbar, con una frecuencia de uso ascendente en los países desarrollados [3]. Por ejemplo, en EE. UU. se realizaron 204.000 cirugías de fusión vertebral en el año 1998, y en el año 2001 este número ascendió a 457.000 [4]. Sin embargo, se ha evidenciado que en algunos pacientes el porcentaje de éxito expresado en términos de dolor controlado y calidad de vida posterior al tratamiento quirúrgico alcanza tan solo un porcentaje de aproximadamente del 30-40\% [5].

En algunos estudios hasta el $20 \%$ de los pacientes con lumbago que cumplen criterios de cronicidad y que son sometidos a manejo quirúrgico pueden llegar a requerir un nuevo procedimiento debido a recurrencias (6). Teniendo en cuenta estos resultados, los pacientes que vayan a ser llevados a artrodesis lumbar requieren de una valoración integral en donde se descarten otras etiologias secundarias que expliquen la cronicidad del dolor, mejorando el pronóstico de funcionalidad y calidad de vida de los pacientes con enfermedad degenerativa vertebral [7].

\section{MATERIALES Y MÉTODOS}

Se desarrolló un estudio descriptivo retrospectivo que tuvo como finalidad determinar qué parámetros espinopélvicos tienen una potencial relación con desenlaces posquirúrgicos favorables en términos de funcionalidad y dolor en los pacientes llevados a artrodesis lumbar. Se seleccionaron 44 pacientes tratados en el son concordantes con lo publicado anteriormente en el área.

Resultados: El mayor cambio porcentual se encontró en el grupo de pacientes que tuvieron una PI-LL > $10^{\circ}$ con un aumento del $50 \%$ en el grupo de pacientes con buena funcionalidad (ODI: 0-20). En SVA > $5 \mathrm{~cm}$, el rango de pacientes con una buena funcionalidad tuvo un aumento del 44,6 \%. El rango de buena funcionalidad en pacientes con una $\mathrm{PT}<20^{\circ}$ aumentó 54,1 puntos porcentuales.

Conclusiones: En la presente cohorte se encontró que los parámetros radiológicos que condicionaron un mayor cambio porcentual en la funcionalidad de los pacientes después de ser llevados a cirugía de columna fueron: SVA de base $>5 \mathrm{~cm}$ y una PI-LL base $>10^{\circ}$ y $\mathrm{PT}<20^{\circ}$.

Palabras clave: Dolor lumbar, artrodesis lumbar, parámetros espinopélvicos, cirugía de columna.

servicio de neurocirugía del Hospital Universitario San Ignacio, Bogotá, Colombia (periodo 01-01-2016-3007-2017).

Los criterios de inclusión fueron: edad > 18 años, dolor lumbar crónico, diagnóstico de canal lumbar estrecho, enfermedad discal degenerativa, espondilolistesis, espondilosis, escoliosis del adulto y/o radiculopatía recurrente. Se excluyeron pacientes < 18 años, con enfermedades congénitas espinales, enfermedades neoplásicas espinales y antecedente de cirugía lumbar con artrodesis.

Se construyó una base datos en Excel para Windows que incluye información sociodemográfica (género, edad y diagnósticos], procedimientos realizados, parámetros imagenológicos: dentro de estos se tomaron incidencia pélvica $(\mathrm{PI})$, inclinación pélvica $(\mathrm{PT})$, inclinación sacra, lordosis lumbar (LL), eje sagital vertical (SVA) y la diferencia entre PI y LL (PI-LL). Para el análisis del balance espinopélvico se tuvo en cuenta los parámetros usados en la literatura internacional (8) y en el servicio de neurocirugía del Hospital Universitario San Ignacio considerando patológica una $\mathrm{PT}>20^{\circ}$, un SVA $>5 \mathrm{~cm}$ y una PI-LL > 10.

Para evaluar los desenlaces se empleó la escala visual analógica (EVA) para clasificar el dolor y se estableció como "dolor controlado" un puntaje $\leq 4$ y "dolor mal controlado" aquel $\geq 5$. La funcionalidad se evaluó mediante la escala Oswestry Disability Index (ODI) [9] categorizando los puntajes de la siguiente manera: discapacidad mínima (0-19), moderada (20-29) y grave (30 o mayor), y variables clínicas concernientes a enfermedad facetaria diagnosticada mediante la maniobra de Acevedo[10]. La maniobra de Acevedo consiste en una fase I, con el paciente acostado en posición de decúbito supino con los miembros inferiores en posición de reposo; la fase II, en la cual se realiza una flexión forzada de la articulación de la cadera, con la pierna extendida y tomando como apoyo la parte más inferior de la pierna, y la fase III, en la que se le pide al 
paciente que realice fuerza hacia abajo y con la mano apoyada en la parte inferior de la pierna. El examinador opone una fuerza opuesta a la anterior, precipitando dolor axial en el segmento lumbar del paciente con disfunción facetaria. Esta prueba es una excelente herramienta clínica no invasiva asequible y de tamizaje de enfermedad facetaria (sensibilidad del $95 \%$ y especificidad del 96 \%) $(10,11)$. Para el seguimiento se realizaron 3 valoraciones por paciente: una evaluación prequirúrgica y otras 2 a los 3 y 6 meses posquirúrgicos. Para el análisis de resultados se empleó el Software STATA v14.0. Posteriormente se realizaron distribución de proporciones y tablas de contingencia para evaluar posibles relaciones entre variables. Las variables continuas se analizaron empleando medidas de tendencia central. El tamaño de la muestra del estudio fue insuficiente para tener resultados estadísticamente significativos.

\section{Parámetros radiológicos espinopélvicos del balance sagital}

A continuación se exponen las definiciones utilizadas para los parámetros estudiados en el presente manuscrito [12] (Figura 1).

SVA: definido como la distancia horizontal desde el plano horizontal de C7 a la esquina posterosuperior del cuerpo vertebral de S1.

PI: el ángulo entre una línea perpendicular al punto medio del platillo vertebral sacro y una línea que conecta este punto al centro de las cabezas femorales.

LL: ángulo entre los planos comprendidos entre el platillo vertebral superior de L1 y el platillo superior de S1.

PT: ángulo entre un plano conectando el centro de las cabezas femorales con el punto medio del platillo vertebral sacro y una línea de referencia vertical desde este punto.

Inclinación sacra: ángulo formado entre una línea de referencia horizontal y una línea paralela al platillo vertebral de S1 (12).

\section{RESULTADOS}

\section{Datos sociodemográficos y valoraciones generales}

Se realizaron análisis de frecuencias para los datos sociodemográficos, encontrando que el 61,3\% fueron mujeres ( $n=27$ ). La edad promedio de cirugía fue 61,1 años (DE = 16 años) (Tabla I). El diagnóstico más frecuente en los pacientes llevados a artrodesis lumbar fue la espondilolistesis (un 20,6\% listesis degenerativa y un $25 \%$ lítica). El 88,5 \% fue llevado a cirugía mínimamente invasiva siendo más frecuente los abordajes intersomáticos por vía anterior (un $34 \%$ de los casos). El $40,9 \%$ de los pacientes $(n=18$ ) tenía en la última valoración clínica prequirúrgica signos de enfermedad facetaria.

En cuanto a dolor en el total de la población, se encontraron las siguientes medianas: en la evaluación prequirúrgica la mediana de puntaje EVA fue de 9 . En la evaluación a los 3 meses una mediana en puntaje EVA

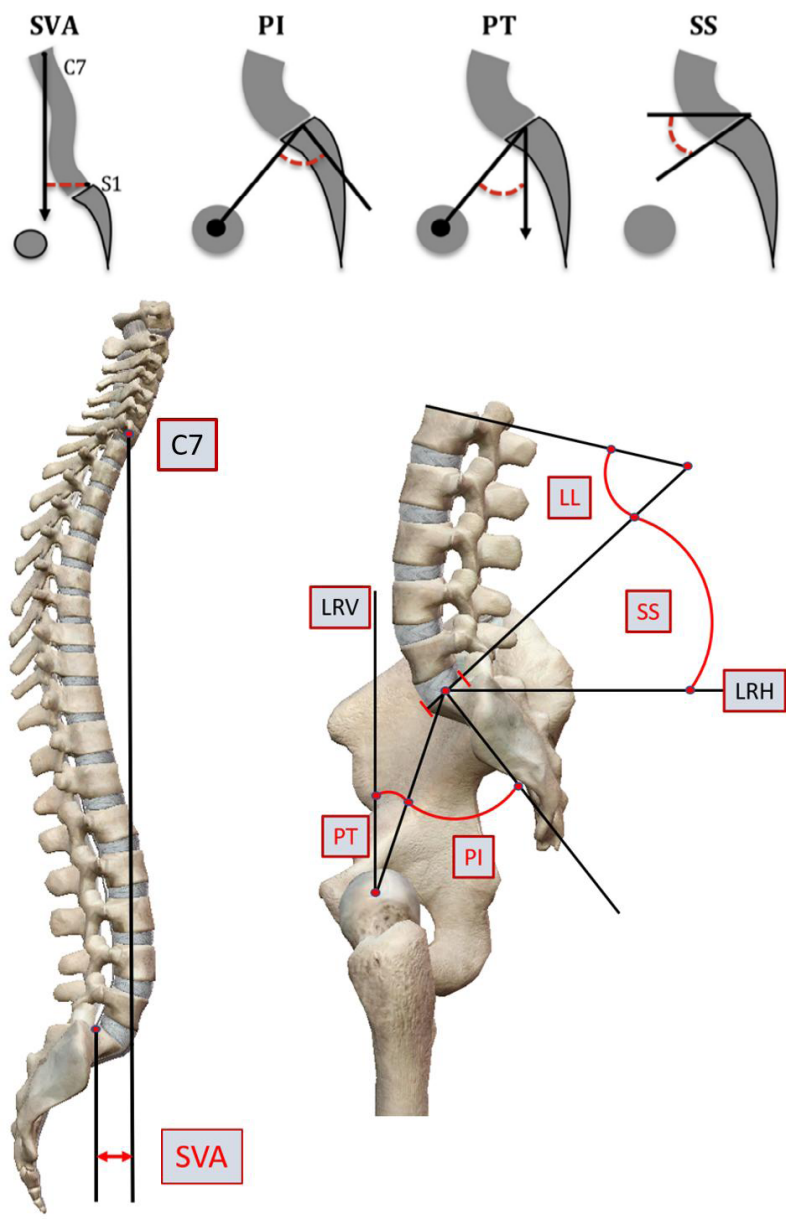

Fig. 1. Parámetros espinopélvicos tradicionales del balance sagital. Arriba se encuentra una vista lateral de la columna vertebral mostrando la técnica de medición del SVA. Imagen tomada de Sardi y cols. (12). Abajo, una imagen lateral de las estructuras espinales, pélvicas y de los miembros inferiores relativas a la medición de los parámetros espinopélvicos. Las abreviaturas de los ángulos son tomadas de la nomenclatura anglosajona: C7: cuerpo vertebral de la séptima vértebra cervical; LRH: línea de referencia horizontal; LRV: línea de referencia vertical; PI: incidencia pélvica; PT: inclinación pélvica; SS: pendiente sacra; SVA: eje vertical sagital.

de 6,5 y, por último, en la evaluación a los 6 meses posquirúrgicos, una mediana en puntaje EVA de 4.

En la evaluación prequirúrgica, los pacientes con "dolor no controlado" (EVA: 5-10) representaron el $100 \%$ en la evaluación, disminuyendo a 70,5 \% a los 3 meses y hasta 48,7\% en la evaluación semestral, es decir una disminución en 51,3 \% al final del seguimiento (Tabla II).

Al analizar la funcionalidad en el total de la población, se encontró que, en la valoración prequirúrgica, únicamente el 22,7\% ( $\mathrm{n}=10$ ) tenía una buena calidad de vida con una discapacidad mínima; este porcentaje se incrementó a los 3 meses a un $63,6 \%(n=28)$ y a 
TABLA I

VARIABLES DEMOGRÁFICAS

\begin{tabular}{|l|c|c|c|}
\hline \multicolumn{4}{|c|}{ Descripción demográfica } \\
\hline \multicolumn{1}{|c|}{ Variable } & Hombre & Mujer & Total \\
\hline Género & $17(38,6 \%)$ & $27(61,3 \%)$ & $44(100 \%)$ \\
\hline Edad promedio (DE) & $57,1(17,4)$ & $63,5(14,8)$ & $61,1(16,0)$ \\
\hline EVA prequirúrgica, mediana & 9 & 10 & 9 \\
\hline EVA posquirúrgica 3 meses, mediana & 6 & 7 & 6,5 \\
\hline EVA posquirúrgica 6 meses, mediana & 2 & 7 & 4 \\
\hline ODI prequirúrgico, mediana & 21 & 24 & 24 \\
\hline ODI posquirúrgico 3 meses, mediana & 13 & 19 & 17 \\
\hline ODI posquirúrgico 6 meses, mediana & 4 & 18 & 11 \\
\hline Enfermedad facetaria & $38,6 \%$ & $66,7 \%$ & $40,9 \%$ \\
\hline
\end{tabular}

DE: desviación estándar. EVA: escala visual analógica. ODI: Oswestry Disability Index.

TABLA II

DESCRIPCIÓN DE LA FUNCIONALIDAD Y EL DOLOR EN LA POBLACIÓN

\begin{tabular}{|c|c|c|c|c|c|c|}
\hline \multicolumn{7}{|c|}{ Dolor EVA } \\
\hline & \multicolumn{2}{|c|}{ Dolor controlado } & \multicolumn{2}{|c|}{ Dolor no controlado } & \multirow{2}{*}{ Total } & \multirow{2}{*}{ Sin datos } \\
\hline Valoración & $n$ & $\%$ & $n$ & $\%$ & & \\
\hline Prequirúrgica & 0 & 0 & 44 & 100 & 44 & 0 \\
\hline 3 meses & 13 & 29,5 & 31 & 70,5 & 44 & 0 \\
\hline 6 meses & 20 & 51,3 & 19 & 48,7 & 39 & 5 \\
\hline \multicolumn{7}{|c|}{ Funcionalidad (ODI) } \\
\hline & \multicolumn{2}{|c|}{$\begin{array}{l}\text { Discapacidad } \\
\text { mínima }\end{array}$} & \multicolumn{2}{|c|}{$\begin{array}{c}\text { Discapacidad } \\
\text { moderada }\end{array}$} & \multicolumn{2}{|c|}{ Discapacidad severa } \\
\hline Valoración & $n$ & $\%$ & $n$ & $\%$ & $n$ & $\%$ \\
\hline Prequirúrgica & 10 & 22,7 & 32 & 72,7 & 2 & 4,6 \\
\hline 3 meses & 30 & 68,2 & 13 & 29,5 & 1 & 2,3 \\
\hline 6 meses & 28 & 71,8 & 10 & 25,6 & 1 & 2,6 \\
\hline
\end{tabular}

EVA: escala visual analógica. ODI: Oswestry Disability Index.

los 6 meses posquirúrgicos a un 69,2\%, presentando un aumento del $49,1 \%$ desde el inicio hasta el final del seguimiento. Se evidenció la inversión de los pacientes que tenían una discapacidad moderada y aquellos con una discapacidad mínima siendo estos la mayoría en el seguimiento semestral (Tabla II).

\section{Evolución del dolor según los parámetros espinopélvicos evaluados}

Tanto los pacientes que tuvieron un SVA mayor o menor a $5 \mathrm{~cm}$ presentaron mejoría en cuanto al dolor pasando de "dolor no controlado" a "dolor controlado", pasando del $0 \%$ al inicio del seguimiento al 51,7\% al final del seguimiento (Tabla III).

La mejoría en el control de dolor de los pacientes con una PT dentro de la normalidad (PT < 20) fue mayor frente a aquellos que tuvieron una PT elevada, teniendo un cambio porcentual del $52,6 \%$ y del $47,4 \%$ respectivamente.

En cuanto al missmatch (PI-LL), se encontró un aumento similar en el control de dolor entre los 2 grupos (patológico vs. no patológico) quienes presentaron una reducción en puntos porcentuales del $51,8 \%$ para aquellos con $\mathrm{PI}-\mathrm{LL}<10^{\circ}$ y una reducción 
TABLA III

EVOLUCIÓN DEL DOLOR CON RESPECTO A LOS PARÁMETROS

\begin{tabular}{|c|c|c|c|c|c|c|c|}
\hline & \multicolumn{6}{|c|}{ Evolución de dolor por parámetros, $n$ [\%] } & \multirow{3}{*}{$\begin{array}{c}\text { Cambio en valores } \\
\text { porcentuales de } \\
\text { control de dolor (pre } \\
\text { vs. } 6 \text { meses] } \\
\text { Dolor controlado }\end{array}$} \\
\hline & \multicolumn{2}{|c|}{ Prequirúrgico } & \multicolumn{2}{|c|}{3 meses } & \multicolumn{2}{|c|}{6 meses } & \\
\hline & Controlado & $\begin{array}{c}\text { No } \\
\text { controlado }\end{array}$ & Controlado & $\begin{array}{c}\text { No } \\
\text { controlado }\end{array}$ & Controlado & $\begin{array}{c}\text { No } \\
\text { controlado }\end{array}$ & \\
\hline \multicolumn{8}{|l|}{ SVA } \\
\hline$<5 \mathrm{~cm}$ & $\mathrm{O}(0)$ & 25 (100) & $6[24]$ & $19[76]$ & $12[57,1]$ & $9(42,9)$ & $+57,1 \%$ \\
\hline$>5 \mathrm{~cm}$ & $0(0)$ & $8(100)$ & $3(37,5)$ & $5(62,5)$ & $4(57,1)$ & $3(42,9)$ & $+57,1 \%$ \\
\hline \multicolumn{8}{|l|}{ PT } \\
\hline$<20^{\circ}$ & $\mathrm{O}(0)$ & $22(100)$ & $8[36,4)$ & $14(63,6)$ & $10(52,6)$ & $9(47,4)$ & $+52,6 \%$ \\
\hline$>20^{\circ}$ & $0(0)$ & $21(100)$ & $4(19,1)$ & $17(80,9)$ & $9(47,4)$ & $10(52,6)$ & $+47,4 \%$ \\
\hline \multicolumn{8}{|l|}{ PI-LL } \\
\hline$<10^{\circ}$ & $0(0)$ & $29(100)$ & $8(27,6)$ & $21(72,4)$ & $14(51,8)$ & $13(48,2)$ & $+51,8 \%$ \\
\hline$>10^{\circ}$ & $\mathrm{O}(0)$ & $15(100)$ & $5(33,3)$ & $10(66,7)$ & $6(50)$ & $6(50)$ & $+50 \%$ \\
\hline \multicolumn{8}{|c|}{ Enfermedad facetaria } \\
\hline Sí & $\mathrm{O}(0)$ & $18(100)$ & $4(22,2)$ & $14(77,8)$ & $10(62,5)$ & $6(37,5)$ & $+62,5 \%$ \\
\hline No & $0(0)$ & $26(100)$ & $9(34,6)$ & $17(65,4)$ & $10(43,5)$ & $13(56,5)$ & $+43,5 \%$ \\
\hline
\end{tabular}

LL: lordosis lumbar. PI-LL: diferencia entre incidencia pélvica y lordosis lumbar. PT: inclinación pélvica. SVA: eje sagital vertical.

del $50 \%$ en los que presentaron un PI-LL patológico (Tabla III).

Los pacientes que presentaron enfermedad facetaria antes de la intervención quirúrgica tuvieron un aumento más prominente en el control de dolor del 62,5\% mientras que aquellos que no tenían enfermedad facetaria y dolor controlado presentaron un aumento del 43,5\% (Tabla III).

\section{Evolución de la funcionalidad según los parámetros evaluados}

Los pacientes que presentaron un SVA $<5 \mathrm{~cm}$ obtuvieron un aumento del $48,2 \%$ en el grupo de discapacidad mínima (del $28 \%$ al $76 \%$ al final del seguimiento) mientras que aquellos con un SVA $>5 \mathrm{~cm}$, es decir patológico, tuvieron un aumento del $44,6 \%$ (del $12,5 \%$ al $57,1 \%$ al final del seguimiento). Sin embargo, se encontró que la reducción de pacientes que presentaron discapacidad severa antes de la cirugía comparado con el seguimiento posquirúrgico fue superior en aquellos que tenían un SVA patológico, reducción del $25 \%$ al $0 \%$ al final de este (Tabla IV).

En cuanto a la relación de la PT con la funcionalidad, se encontró que aquellos con una PT no patológica presentaron un mayor aumento en el grupo de pacientes con discapacidad mínima del 54,1 \% aunque también presentaron un aumento del $6 \%$ en aquellos con discapacidad severa. Los pacientes que presentaron una PT patológica registraron un aumento favorable del 40,3\% en el grupo de discapacidad mínima con una disminución del $9,5 \%$ al $0 \%$ en aquellos con discapacidad severa.

Entre los pacientes que obtuvieron resultados patológicos en la PI-LL tuvieron un aumento mayor de pacientes con discapacidad mínima de 50 puntos porcentuales, pasando del $33,3 \%$ al $83,3 \%$ a los 6 meses; y además presentaron una reducción del $13,4 \%$ en aquellos con discapacidad severa al final del seguimiento.

Los resultados relacionados con enfermedad facetaria presentaron comportamientos contrarios frente a los resultados de los demás parámetros. Aquellos con enfermedad facetaria tuvieron un aumento de 46,6 puntos porcentuales (del 22,2 \% al 68,8 \%) en los pacientes con discapacidad mínima pero también un aumento del 6,9\% en la discapacidad severa (del $5,6 \%$ al $12,5 \%$ ). Los pacientes que no presentaron enfermedad facetaria registraron una disminución del grupo de discapacidad severa del $7,7 \%$ así como un aumento del $46,5 \%$ en aquellos con discapacidad mínima (del 23,1\% al 69,6\%) (Tabla IV).

La Tabla $\vee$ resume las cifras de cambio en términos de puntos porcentuales antes de la intervención quirúrgica y al final del seguimiento. Es importante resaltar que la reducción en el grupo de discapacidad severa se encontró siempre en las mediciones patológicas de los parámetros, de hecho, la mayor disminución fue de 25 puntos porcentuales en el caso del SVA, de 13,4 puntos porcentuales en la PI-LL, de 9,5 puntos porcentuales en la PT y de 7,7 puntos porcentuales en la enfermedad facetaria. 
TABLA IV

EVOLUCIÓN DE LA FUNCIONALIDAD SEGÚN PARÁMETROS ESPINO PÉLVICOS

\begin{tabular}{|c|c|c|c|c|c|c|c|c|c|c|c|c|}
\hline & \multicolumn{9}{|c|}{ Evolución de la funcionalidad por parámetros, n [\%] } & \multirow{2}{*}{\multicolumn{3}{|c|}{ Cambio en valores porcentuales en funcionalidac }} \\
\hline & \multicolumn{3}{|c|}{ Prequirúrgico } & \multicolumn{3}{|c|}{3 meses } & \multicolumn{3}{|c|}{6 meses } & & & \\
\hline & $\begin{array}{l}\text { Discapacidad } \\
\text { mínima }\end{array}$ & $\begin{array}{c}\text { Discapacidad } \\
\text { moderada }\end{array}$ & $\begin{array}{c}\text { Discapacidad } \\
\text { severa }\end{array}$ & $\begin{array}{c}\text { Discapacidad } \\
\text { mínima }\end{array}$ & $\begin{array}{c}\text { Discapacidad } \\
\text { moderada }\end{array}$ & $\begin{array}{c}\text { Discapacidad } \\
\text { severa }\end{array}$ & $\begin{array}{l}\text { Discapacidad } \\
\text { mínima }\end{array}$ & $\begin{array}{c}\text { Discapacidad } \\
\text { moderada }\end{array}$ & $\begin{array}{c}\text { Discapacidad. } \\
\text { severa }\end{array}$ & $\begin{array}{l}\text { Discapacidad } \\
\text { mínima }\end{array}$ & $\begin{array}{c}\text { Discapacidad } \\
\text { moderada }\end{array}$ & $\begin{array}{c}\text { Discapacidad } \\
\text { severa }\end{array}$ \\
\hline \multicolumn{13}{|l|}{ SVA } \\
\hline$<5 \mathrm{~cm}$ & $7(28)$ & $17(68)$ & $1(4)$ & $17(64)$ & 8 (32) & $1(4)$ & $16(76,2)$ & $4(19,1)$ & $1(4,7)$ & $+48,2 \%$ & $-48,9 \%$ & $+0,7 \%$ \\
\hline$>5 \mathrm{~cm}$ & $1(12,5)$ & $5(62,5)$ & 2 (25) & $4(50)$ & $4(50)$ & $0(0)$ & $4(57,1)$ & $3(42,9)$ & $\mathrm{O}(0)$ & $+44,6 \%$ & $-19,6 \%$ & $-25 \%$ \\
\hline \multicolumn{13}{|l|}{ PT } \\
\hline$<20^{\circ}$ & $2(9,1)$ & $19(86,4)$ & $1(4,5)$ & $15(68,2)$ & $6(27,3)$ & $1(4,5)$ & $12(63,2)$ & $5(26,3)$ & $2(10,5)$ & $+54,1 \%$ & $-60,1 \%$ & $+6 \%$ \\
\hline$>20^{\circ}$ & $7(33,3)$ & $12(57,2)$ & $2(9,5)$ & $12(57,1)$ & $9(42,9)$ & $\mathrm{O}(0)$ & $14(73,6)$ & $5(26,3)$ & $\mathrm{O}(0)$ & $+40,3 \%$ & $-30,9 \%$ & $-9,5 \%$ \\
\hline \multicolumn{13}{|l|}{ PI-LL } \\
\hline$<10^{\circ}$ & $5(17,2)$ & $23(79,3)$ & $1(3,5)$ & $19(65,5)$ & $9(31)$ & $1(3,5)$ & $17(63)$ & $8(29,6)$ & $2(7,4)$ & $+45,8 \%$ & $-49,7 \%$ & $+3,9 \%$ \\
\hline$>10^{\circ}$ & $5(33,3)$ & $8(53,3)$ & $2(13,4)$ & $9(60)$ & $6(40)$ & $0(0)$ & $10(83,3)$ & $2(16,7)$ & $\mathrm{O}(0)$ & $+50 \%$ & $-36,6 \%$ & $-13,4 \%$ \\
\hline \multicolumn{13}{|c|}{ Enfermedad facetaria } \\
\hline Sí & $4(22,2)$ & 13 (72,2) & $1(5,6)$ & $14(77,8)$ & $3(16,7)$ & $1(5,5)$ & $11(68,8)$ & $3(18,7)$ & $2(12,5)$ & $+46,6 \%$ & $-53,5 \%$ & $+6,9 \%$ \\
\hline No & $6(23,1)$ & $18(69,2)$ & $2(7,7)$ & $14(53,9)$ & $12(46,1)$ & $\mathrm{O}(0)$ & $16(69,6)$ & $7(30,4)$ & $\mathrm{O}(0)$ & $+46,5 \%$ & $-38,8 \%$ & $-7,7 \%$ \\
\hline
\end{tabular}

LL: lordosis lumbar. PI-LL: diferencia entre incidencia pélvica y lordosis lumbar. PT: inclinación pélvica. SVA: eje sagital vertical. 
TABLA V

RESUMEN DE CAMBIO EN TÉRMINOS DE PUNTOS PORCENTUALES DE DOLOR Y FUNCIONALIDAD Cambio porcentual en el seguimiento (pre. vs. 6 meses)

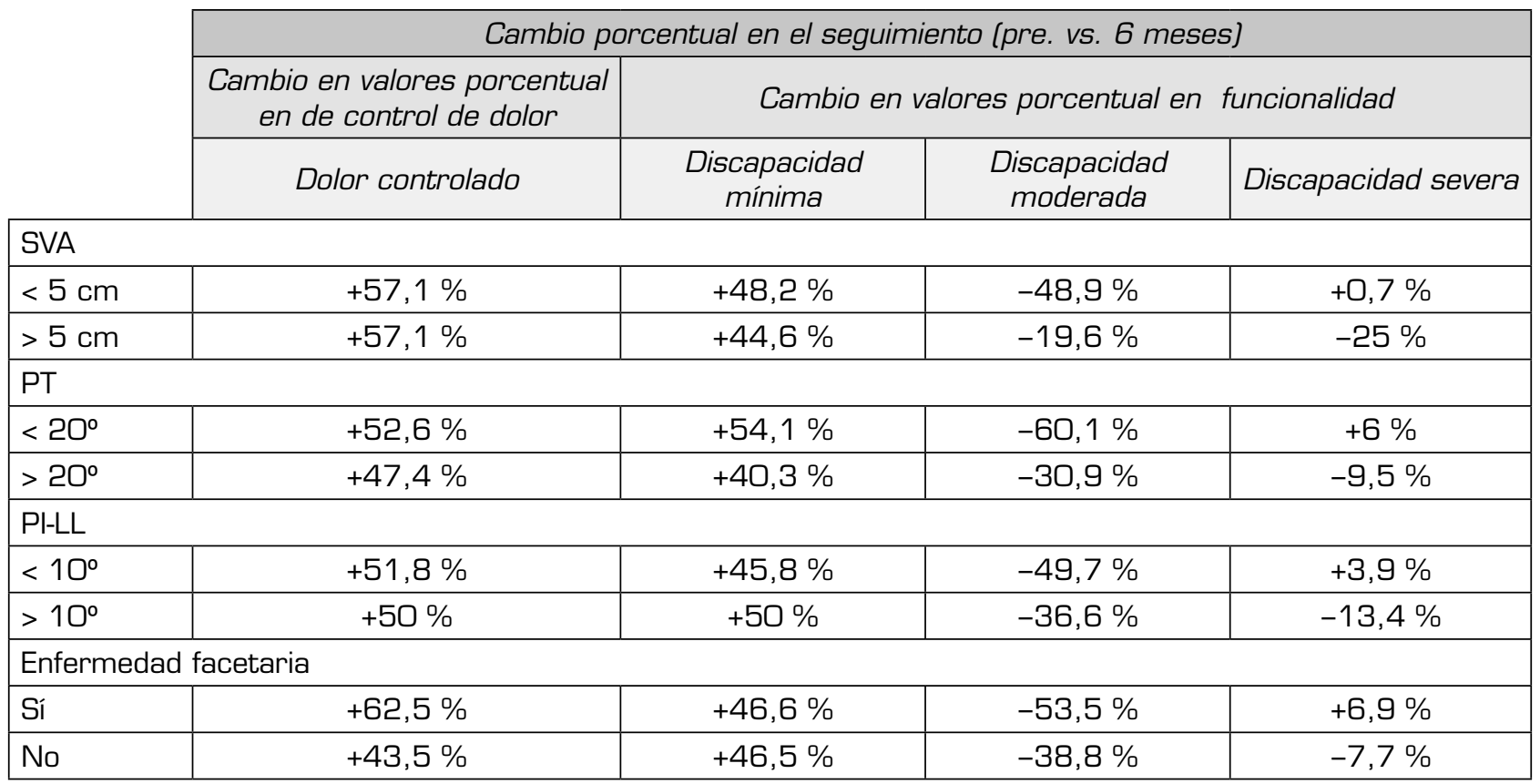

LL: Iordosis lumbar. PI-LL: diferencia entre incidencia pélvica y lordosis lumbar. PT: inclinación pélvica. SVA: eje sagital vertical.

\section{DISCUSIÓN}

La presente cohorte encontró que las mediciones de dolor controlado tuvieron un impacto más contundente después de la cirugía cuando los parámetros evaluados se encontraron dentro de la normalidad antes de la misma. Sin embargo, en las mediciones de funcionalidad se halló un comportamiento más complejo, encontrando que los aumentos en grupos de discapacidad mínima siempre fueron mayores cuando estos se encontraron en rangos de normalidad, aunque la disminución en los grupos de discapacidad severa siempre fue mayor cuando los parámetros estaban en rangos patológicos antes de la cirugía. Es decir, los beneficios netos de la cirugía para pacientes con discapacidad severa son más contundentes cuando los parámetros se encuentran elevados (por ejemplo, SVA, PI-LL, PT).

En la literatura se ha encontrado que la PT y el desbalance entre la PI y LL (mismatch) son variables importantes como predictores de éxito en pacientes llevados a artrodesis lumbar (13) (tanto para corrección de deformidad sagital y coronal (14) como los casos llevados a cirugía mínimamente invasiva) (15). En 2016 , Than y cols. (15) realizaron un estudio retrospectivo en el que llevaron a cabo un seguimiento de 2 años posquirúrgicos a 104 pacientes a los que se dividió en 2 grupos: aquellos que representaron el $20 \%$ superior en términos de cambio en escalas ODI y EVA, y aquellos pacientes con el $20 \%$ menor en dichos parámetros. De esta manera se encontró que aquellos que tenían un ODI mayor prequirúrgico se beneficiaban más de la intervención quirúrgica. Ahora bien, dentro de sus resultados no encontraron diferencias estadísticamente significativas en cuanto a los parámetros espinopélvicos prequirúrgicos, sin embargo, mencionan que los pacientes con mejores resultados tuvieron SVA, PI-LL y PT menores a los que tuvieron peores resultados quirúrgicos. Estos resultados irían en concordancia con los presentados en este estudio en donde se puede apreciar que en los pacientes que tuvieron el mayor cambio porcentual se encontraron mediciones SVA y PI-LL alterados en sus valoraciones prequirúrgicos.

En otro estudio realizado por Smith y cols. (16) se encontró como predictor de peores desenlaces en términos de ODI mayor índice de masa corporal de base y mayor SVA al final del seguimiento. Dentro de sus hallazgos, un ODI más afectado antes de la cirugía es predictor de una mayor mejoría funcional en el seguimiento, resultado similar al que se obtuvo en esta ocasión. Sin embargo, en nuestra cohorte los pacientes con una funcionalidad más disminuida se mantuvieron relativamente estable, disminuyendo del 4,5\% al $2,6 \%$, que, si bien supone una disminución de $57 \%$, no representa un cambio comparado con el comportamiento de los pacientes con funcionalidad buena y moderada, este efecto es posible atribuirlo al efecto suelo que sería inherente a su discapacidad.

\section{CONCLUSIONES}

Se encontró que los parámetros radiológicos que condicionaron un mayor cambio porcentual en la funcionalidad de los pacientes con discapacidad severa 
fueron: SVA $>5 \mathrm{~cm}$ y una PI-LL base $>10^{\circ}$. Sin embargo, las mediciones de dolor controlado (O-4 en EVA) tuvieron unos aumentos mayores en los pacientes con mediciones de SVA y PI-LL normales. Asimismo, se encontró una posible asociación en aquellos pacientes que presentan enfermedad facetaria antes de ser llevados a cirugía y mejores desenlaces postoperatorios. Dentro de la práctica clínica, es esencial estandarizar la toma de ortorradiografía en el postoperatorio para poder evaluar en qué grado se modifican los parámetros espinopélvicos que se analizaron en el estudio y poder llegar a correlacionarlos con el tipo de intervención quirúrgica realizada en futuras investigaciones para así aumentar la evidencia para tener resultados estadísticamente significativos.

\section{CONFLICTO DE INTERESES}

Los autores declaran no tener ningún conflicto de intereses.

\section{BIBLIOGRAFÍA}

1. Last AR, Hulbert K. Chronic low back pain: Evaluation and management. Am Fam Physician. 2009;79(12):1067-74.

2. Hoy D, Brooks P, Blyth F, Buchbinder R. The epidemiology of low back pain. Best Pract Res Clin Rheumatol [Internet]. 2010;24(6):769-81. DOI: 10.1016/j.berh.2010.10.002.

3. Berbeo M, Diaz R, Perez JC, Giraldo-Grueso M, Gutierrez $S$, Villegas $M C$, et al. Minimally invasive surgical approach for spinal canal tumors-Technique description and experience from a reference center. J Cancer Ther [Internet]. 2017;8(3):268-77. DOI: 10.4236/jct.2017.83022.

4. Von Korff M, Crane P, Lane M, Miglioretti DL, Simon G, Saunders K, et al. Chronic spinal pain and physical-mental comorbidity in the United States: Results from the national comorbidity survey replication. Pain. 2005;113(3):331-9. DOI: 10.1016/j.pain.2004.11.010.

5. Salvetti MDG, Pimenta CADM, Braga PE, Corrêa CF. Disability related to chronic low back pain: Prevalence and associated factors. Rev Esc Enferm USP [Internet]. 2012;46 Spec:1623. DOI: 10.1590/S0080-62342012000700003.

6. Adams MA. Biomechanics of back pain. Acupunct Med. 2004;22(4):178-88. DOI: 10.1136/aim.22.4.178.
7. Deyo RA, Weinstein JN. Low back pain. N Engl J Med [Internet]. 2001;344(5):363-70. DOI: 10.1056/ NEJM200102013440508.

8. Ames CP, Smith JS, Scheer JK, Bess S, Bederman SS, Deviren $\mathrm{V}$, et al. Impact of spinopelvic alignment on decision making in deformity surgery in adults. J Neurosurg Spine [Internet]. 2012;16(6):547-64. DOI: 10.3171/2012.2.SPINE11320.

9. Payares K, Lugo LH, Morales V, Londoño A. Validation in Colombia of the Oswestry disability questionnaire in patients with low back pain. Spine (Phila Pa 1976). 2011;36(26):E17305. DOI: 10.1097/BRS.Ob013e318219d184.

10. Acevedo J. Síndrome facetario lumbar. Nuevo signo de diagnóstico clínico. Rehabilitación [Internet]. 2004;38(4):16874. doi: 10.1016/S0048-7120(04)73452-0. Disponible en: http://www. elsevier.es/es-revista-rehabilitacion-120-articulo-sindrome-facetario-lumbar-nuevo-signo-13064724

11. Lindado Pacheco CA, Gutierrez S, Acevedo González JC. Factores pronósticos para artrodesis lumbar. Univ Médica [Internet]. 2018;60(1):1-8. DOI: 10.11144/Javeriana. umed60-1.artr.

12. Sardi JP, Camacho JE, Diaz RC, Berbeo ME. The Berbeo-Sardi Angle (BSA): An innovative method to effectively estimate pelvic retroversion in anteroposterior radiographs-A correlation with traditional parameters. Spine Deform [Internet]. 2017;6(2):105-11. DOl: 10.1016/j.jspd.2017.08.011.

13. Matsumoto T, Okuda S, Maeno T, Yamashita T, Yamasaki R, Sugiura $T$, et al. Spinopelvic sagittal imbalance as a risk factor for adjacent-segment disease after single-segment posterior lumbar interbody fusion. J Neurosurg Spine [Internet]. 2017;26(4):435-40. DOI: 10.3171/2016.9.SPINE16232.

14. Lamartina C, Berjano P, Petruzzi M, Sinigaglia A, Casero G, Cecchinato $\mathrm{R}$, et al. Criteria to restore the sagittal balance in deformity and degenerative spondylolisthesis. Eur Spine J. 2012; 21 Suppl 1:S27-31. DOI: 10.1007/s00586-0122236-9.

15. Than KD, Park P, Fu K, Nguyen S, Wang MY, Chou D, et al. Clinical and radiographic parameters associated with best versus worst clinical outcomes in minimally invasive spinal deformity surgery. J Neurosurg Spine. 2016;25(1):21-5. DOI: 10.3171/2015.12.SPINE15999.

16. Smith JS, Shaffrey Cl, Lafage V, Schwab F, Scheer JK, Protopsaltis $T$, et al. Comparison of best versus worst clinical outcomes for adult spinal deformity surgery: a retrospective review of a prospectively collected, multicenter database with 2-year follow-up. J Neurosurg Spine [Internet]. 2015;23(3):34959. DOI: 10.3171/2014.12.SPINE14777. 